\title{
An atypical case of drug-induced lupus syndrome
}

\begin{abstract}
DIL is an autoimmune vasculitis against certain drugs that lead to autoantibodies in some patients causing a clinical syndrome with similar clinical manifestations of SLE. A 30 year old female was admitted for dry cough, polyarthralgia, and subfebrile fever that arouse three weeks after termination of antibiotic treatment for maxillary sinusitis. Computed tomography revealed multiple subpleural nodules. BAL culture was negative for bacteria, fungus, and ARB. Histopathologic examination of the TBB specimen revealed granulomatous inflammation. ANA, P-ANCA, MPO-ANCA, PR3-ANCA was positive while anti-ds-DNA, C-ANCA, C3 and C4 were negative. Anti-histone antibody was high. Symptoms resolved within three months after methylprednisolone treatment. Final diagnosis was DIL due to amoxicillin clavulanate. Certain drugs may trigger an autoimmune response causing DIL that has similarities to SLE with crucial differences in clinical and immunologic features. Our case was a diagnostic challenge due to its atypical presentation with significant overlap of the clinical and laboratory findings.
\end{abstract}

Keywords: drug-induced lupus, SLE, DIL, drug-induced vasculitis, lupus-like syndrome, amoxicillin-clavulanate
Volume 8 Issue 4 - 2018

\author{
Cuneyt Tetikkurt,' Seza Tetikkurt² \\ 'Department of Pulmonary Medicine, Istanbul University, Turkey \\ ${ }^{2}$ Department of Pathology, Bağcilar Training and Research \\ Hospital, University of Health Sciences, Turkey
}

\begin{abstract}
Correspondence: Cuneyt Tetikkurt, Department of Pulmonary Medicine, Cerrahpasa Medical Faculty, Istanbul University, Turkey,
\end{abstract} Tel +90-216-36019 77, Email tetikkurt@gmail.com

Received: August 03, 2018| Published: August 29, 2018

\section{Introduction}

Certain drugs may trigger an autoimmune response that induce autoantibodies which may cause a clinical syndrome with features similar to systemic lupus erythematosus in some patients, termed drug-induced lupus. These adverse reactions include fever, rash, leucopenia, arthiritis, vasculitis, pulmonary manifestations, and symptoms of SLE [Systemic Lupus Erythematosus (SLE)]. DIL [Drug-Induced Lupus (DIL)] although akin to SLE reveals notable differences in clinical and autoimmune features. ${ }^{1,2}$ Pulmonary complications like interstitial pneumonia, adult respiratory distresslike syndrome, and pleural effusion are extremely rare. ${ }^{3-5}$ DIL is now well established and many other autoimmune disorders including vasculitis may arise as a drug reaction. Most of the cases of antibiotic induced autoimmune phenomena are due to vasculitis. Mortality rate of DIL is low and prognosis is excellent once the drug is discontinued although rare significant complications may come out due to systemic manifestations. The rate of acetylation is genetically predetermined and subjects with slow acetylation rates have a higher incidence of DIL. ${ }^{6,7}$ Acetylation rate is the hallmark of DIL that determines the prognostic outcome or the severity of the clinical profile.

We present the case of a patient who developed subfebrile fever, subpleural nodules, and mediastinal lymph node enlargement following amoxicillin-clavunate treatment.There are fundamental differences in the clinical features of DIL and vasculitis that may allow an accurate diagnosis in most of the patients but the distinction was not easily achievable in our case due to the significant overlap of clinical and laboratory manifestations with an atypical clinical profile. The clinicians should be aware of the variable and atypical clinical profile of DIL that may cause a diagnostic confusion leading to a severe prognostic outcome if the offending agent is not withdrawn promptly.

\section{Case report}

A 30 year old woman was admitted for dry cough, polyarthralgia, and subfebrile fever that developed following two weeks of amoxicillin-clavulanate treatment for maxillary sinusitis. Her past medical history was remarkable. The family history did not reveal any disease of medical interest. Four weeks before admission the patient developed fever $\left(38.4^{\circ} \mathrm{C}\right)$, postnasal purulent discharge, and maxillary pain. WBC was $13.2 \times 103 / \mathrm{ml}$ with 79 percent neutrophils, CRP: 55.5, ERS: $48 \mathrm{~mm} / \mathrm{h}$. The patient was commenced on amoxicillin clavulanate $1000 \mathrm{mg}$ bid for fourteen days. Dry cough, dyspnea on exertion, polyarthralgia, and fever occured three weeks after amoxicillin clavulanate treatment. Chest x-ray was normal (Figure 1). Blood biochemistry was within normal limits. There was no radiologic abnormalitiy on joint films. Arterial blood gases; $\mathrm{pH}$ : $7.39, \mathrm{pO}_{2}: 84.2$, and $\mathrm{pCO}_{2}: 38.6 \mathrm{~mm} \mathrm{Hg}$. ECG revealed sinus rhytm $(84 / \mathrm{min})$. Tuberculin test was negative. Chest CT showed bilateral multiple subpleural nodules in the lingula (one; $3 \mathrm{~mm}$ ), left lower lobe (multiple; $1-2 \mathrm{~mm}$ ), right lobe anterior segment (two; $5 \mathrm{~mm}$ and $15 \mathrm{X} 10 \mathrm{~mm}$ ) (Figure 2). Serum ACE was 24U/L. Sputum smear and culture were negative for bacteria, mycobacteria, and fungus.

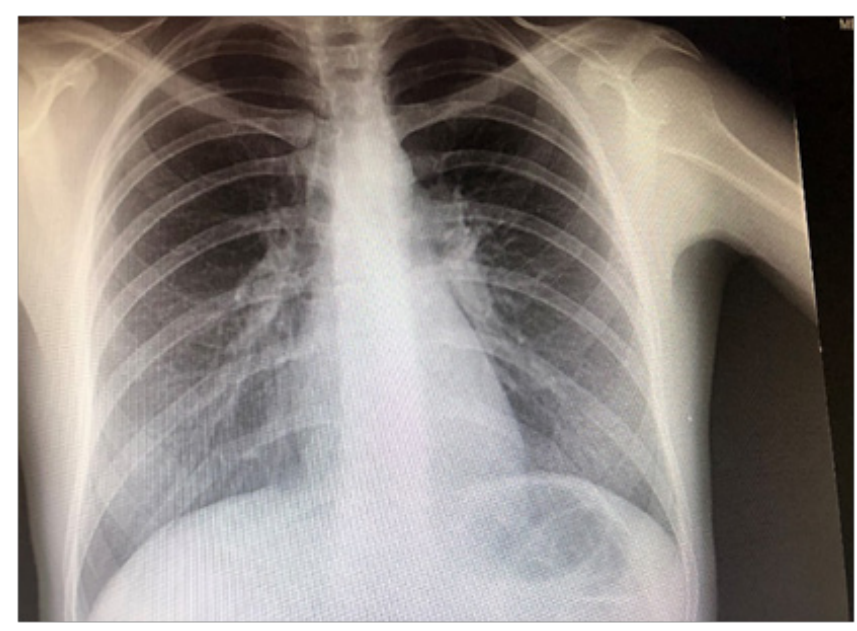

Figure I Normal initial chest x-ray. 


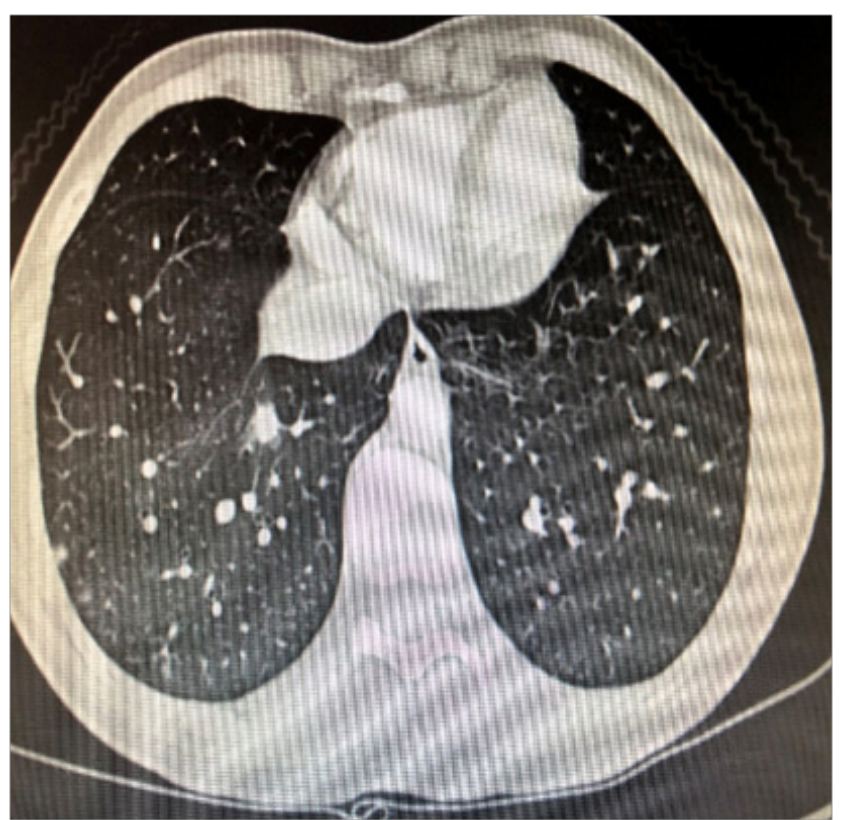

Figure 2 Chest CT revealing pulmonary nodules.

Serologic tests including rheumatoid factor, anti-doublestranded deoxyribonucleic acid, complement levels, antiglomerular basal membrane antibody, and Brucella agglutination test were negative. Serum antinuclear antibodies (ANA: ++, homogeneous), perinuclear anti-neutrophil cytoplasmic antibodies (P-ANCA: +), cytoplasmic anti-neutrophil cytoplasmic antibodies (C-ANCA: ++ ), antimyeloperoxidase antibodies (MPO-ANCA: 98.4EU/ml), and antiproteinase 3 antibodies (PR3-ANCA: 34.62EU/ml) were positive. BAL revealed a normal total and differential cell count. Culture of the BAL fluid was negative for bacteria, tuberculosis, and fungus. Histopathologic examination of the transbronchial specimen did not demonstrate any features of vasculitis but revealed non-caseating granulomatous inflammation. Serum anti-histone antibody was 4.2units. Final diagnosis was drug-induced-lupus due to amoxicillinclavulanate and methylprednisolone $(48 \mathrm{mg} /$ day) was commenced. Symptoms, laboratory, and radiologic findings completely resolved within three months of steroid treatment. The patient showed an uneventful recovery following six months of steroid treatment.

\section{Discussion}

It has long been known that many drugs, including antibiotics can induce DIL or vasculitis. ${ }^{1,3}$ Patients can be designated as DIL or vasculitis according to the accepted definitions, clinical, and serological findings. We present a case that presented with an atypical clinical picture of vasculitis following amoxicilline-clavunate treatment for maxillay sinusitis revealing overlapping manifestations of DIL and vasculitis $^{5,6}$ due to amoxicillin-clavulanate. The final diagnosis was DIL due to positive anti-histone antibody. After steroid treatment, the patient showed an outstanding outcome with resolution of symptoms, clinical, laboratory, and radiologic findings. The patient was a diagnostic challenge for the clinician because the risk of developing DIL with amoxicillin-clavunate is extremely rare. ${ }^{4,6,7}$ Moreover, the distinction between DIL and vasculitis was not achievable due to the the significant overlap of the clinical manifestations between these two entities in our case. DIL patients present with various manifestations like arthralgia, pericarditis, pleuritis, and fever. Dry cough, subfebrile fever, and polyarthralgia were the predominant symptoms in our case. Chest CT revealed pulmonary nodules and enlarged mediastinal lymph nodes that are unusual in DIL. And thirdly, the clinical manifestations were atypical for DIL. Notably the presence of subpleural nodules and the enlarged mediastinal lymph nodes identified by chest CT lead to diagnostic confusion.

Aloush has reported crucial differences between the DIL and ANCA-associated vasculitis by reviewing their alikeness and discrepancies. DIL patients have more musculoskeletal complaints, more serositis, and gastrointestinal involvement while the ANCAassociated vasculitis more frequently presents with pulmonary and renal involvement. ANA, anti-DNA, and anti-histone antibodies are prevalant in DIL, p-ANCA is found in a similar proportion of patients in both groups whereas c-ANCA is detected only in patients with vasculitis. DIL patients usually recover completely after drug withdrawal while patients with vasculitis need steroids or immunosuppressive drugs frequently. ${ }^{8}$ Steroid treatment was needed because of the persistent clinical profile of our patient. There was also significant overlap of laboratory findings of DIL and vasculitis in this case. Antinuclear antibodies are positive in over $50 \%$ of cases and native anti-DNA is reported. ${ }^{9}$ These markers were negative. Resolution of symptoms, positive serum anti-histone antibody with positive c-ANCA, and complete resolution radiologic findings after drug withdrawal strongly suggested DIL due to amoxicllinclavulanate treatment.

Some patients with DIL develop a much more serious picture of adverse autoimmune response characterized by high titres of antibodies to MPO with clinical manifestations including pauciimmune necrotizing and crescentic glomeruloneprithis, upper respiratory tract disease, and pulmonary hemorrhage with negative or low titres of ANA. ${ }^{10,11}$ There were no signs of MPO-ANCA vasculitis with alveolar hemorrhage and microhematuria supporting the presence of alveolar and glomerular inflammation as Bosch has suggested. ${ }^{12,13}$ DIL usually occurs in a few weeks of treatment but may develop after months or years. It is not usually dose dependent and frequently improves after drug withdrawal. ${ }^{1,3,4}$ The symptoms appeared two weeks after antibiotic treatment was completed in our patient. Antibodies to double-stranded DNA were absent and complement levels were normal. The PR3-ANCA and MPO-ANCA serum levels decreased in correlation with the course of symptoms and the resolution of radiologic findings after antibiotic treatment.

The pathogenesis of drug-induced lupus is uncertain. However, many different theories exist for the pathogenesis of drug-induced lupus. ${ }^{2}$ The predominant pathogenetic mechanisms that influence the risk of DIL occurence are genetic differences in drug metabolism such as the acetylator status and the immunogenetic characteristics of the patient. Possible genetic risk factors include human leukocyte antigen (HLA)-DR4, HLA-DR0301, and the complement C4 null allele. These factors vary between different agents. ${ }^{7,14-16}$ The mechanism responsible for ANCA and vasculitis in patients on amoxicillin clavulanate is unknown. ANCA production may occur due to the interaction between amoxicillin clavulanate and neutrophils or neutrophil MPO. The reactive intermediates that are activated by MPO and hydrogen preoxide are immunogenic for T-cells and stimulate the immune system. ${ }^{17,18}$ The cytotoxic activity of the metabolites determine the cell death and production of autoantibodies. ${ }^{19}$ Vasculitic symptoms were not present in our case but systemic manifestations required steroid 
treatment. The amoxicillin clavulanate associated vasculitis or DIL is rare. It is important to be aware of this complication because early withdrawal of the drug results in clinical recovery and may prevent a fatal outcome. Early recognition of this syndrome is an extremely important point because prompt drug withdrawal eventuates in clinical recovery and prevents severe complications.

Respiratory and systemic manifestations were preliminary in our patient. The clinical findings and the serologic markers were inadequate for exact DIL, ANCA-associated vasculitis or collagen vascular disease diagnosis. Resolution of symptoms together with normalization thelung nodules and the enlarged mediastinal lymph nodes after steroid treatment strongly suggests drug induced autoimmune disease. Genetic factors, immunogenetics, acelytor staus, DNA methyltransfrease activity, and autoantibodies including antinuclear, anti-histone, and antineutrophil cytoplasmic antibodies may play a role in the pathogenesis of DIL. ${ }^{6,-12}$ The complex pathogenetic mechanism of DIL leads to a variable patient profile concerning the symptoms, laboratory, and radiologic manifestations. Consequently, different presentations, overlap syndromes, atypical clinical, and laboratory findings associated with drug-induced disease represent a diagnostic challenge for the pulmonary clinician. The current case does not fit into any of the previously designated classifications.

\section{Conclusion}

The pathogenetic mechanisms of DIL remain uncertain and may lead to variable manifestations between different drugs. Patient characteristics may influence risk of DIL and define the clinical profile including the laboratory and the radiologic findings. Genetic factors, immunogenetics, acelytor status, DNA methyltransferase activity, and different autoantibodies lead to a complex pathogenetic DIL pattern. This complex pathogenetic mechanism is predominantly liable for the diversity of the patient's clinical profile. Our patient presented with atypical clinical manifestations, laboratory, and radiologic findings that were neither compatible with DIL nor with vasculitis. The only clue to DIL diagnosis was the high serum antihistone level. Clinicians should bear in mind that the identification of DIL and distinction from vasculitis may not be easily achievable due to the atypical patient symptoms, overlapping clinical, and laboratory manifestations. Consequently, we believe that drug-induced vasculitis would be a better term to designate drug-induced lupus like vasculitic or autoimmune disorders.

\section{Acknowledgements}

None.

\section{Conflict of interest}

Cuneyt Tetikkurt, Seza Tetikkurt do not have any conflicts of interests to declare associated with this case report.

\section{References}

1. Yung RL, Richardson BC. Drug-induced lupus. Rheum Dis Clin North
Am. 1994;20(1):61-86.

2. Rubin RL. Drug induced lupus. Toxicology. 2005;209(2):135-147.

3. Hess E. Drug-related lupus. N Engl J Med. 1988;318:1460-1462.

4. Katz U, Zandman-Goddard G. Drug-induced lupus: an update. Autoimmun Rev. 2010;10(1):46-50.

5. Diri E, Tello W, Ratnoff WD. Nugent K.Infliximab-induced SLE-like syndrome involving the lung and pleura. Lupus. 2007;16(9):764-766.

6. Rubin RL. Etiology and mechanisms of drug-induced lupus. Curr Opin Rheumatol. 1999;11(5):357-363.

7. Vasoo S. Drug-induced lupus: an update. Lupus. 2006;15(11):757-761.

8. Aloush V, Litinsky I, Caspi D, et al. Propylthiouracil-induced autoimmune syndromes: two distinct clinical presentations with different course and management. Semin Arthirits Rheum. 2006;36(1):4-9.

9. Dolman KM, Gans RO, Vervaat TJ, et al. Goldschmeding R. Vasculitis and antineutrophil cytoplasmic autoantibodies associated with prophylthiouracil therapy. Lancet. 1993;342(8872):651-652.

10. D'Cruz D, Chesser AM, Lightowler C, et al. Antineutrophil cytoplasmic antibody-positive crescentic glomerulonephritis associated with antithyroid drug treatment. Br J Rheumatol. 1995;34(11):1090-1091.

11. Cambridge G, Wallace H, Bernstein RM, Leaker B. Autoantibodies to myeloperoxidase in idiopathic and drug-induced systemic lupus erithematosus and vasculitis. Br J Rheumatol. 1994;33(2):109-114.

12. Bosch X, López-Soto A, Mirapeix E, et al. Anti-neutrophil cytoplasmic autoantibody-associated alveolar capillaritis in patients presenting with pulmonary hemorrhage. Arch Pathol Lab Med. 1994;118:517-522.

13. von Schmiedeberg S, Goebel C, Gleichmann E, Uetrecht J. Neutrophils and drug metabolism. Science. 1995;268(5210):585-586.

14. Batchelor JR, Welsh KI, Tinoco RM, et al. Hydralazine-induced systemic lupus erythematosus: influence of HLA-DR and sex on susceptibility. Lancet. 1980;1(8178):1107-1109.

15. Speirs C, Fielder AH, Chapel H, et al. Complement system protein $\mathrm{C} 4$ and susceptibility to hydralazine-induced systemic lupus erythematosus. Lancet. 1989;333(8644):922-924.

16. Jeffries M, Bruner G, Glenn S, et al. Sulpha allergy in lupus patients: a clinical perspective. Lupus. 2008;17(3):202-205.

17. Gunnarsson I, Nordmark B, Hassan Bakri A, et al. Development of lupus-related side-effects in patients with early RA during sulphasalazine treatment-the role of IL-10 and HLA. Rheumatology (Oxford). 2000;39(8):886-893.

18. Cambridge G, Wallace H, Bernstein RM, Leaker B. Autoantibodies to myeloperoxidase in idiopathic and drug-induced systemic lupus erithematosus and vasculitis. Br J Rheumatol. 1994;33(2):109-114.

19. Kimura S, Ikeda-Saito M. Human myeloperoxidase and thyroid peroxidase, two enzymes with separate and distinct physiological functions, are evolutionary related members of the same gene family. Proteins. 1998;3(2):113-120. 